\title{
Production of quarkonia and heavy flavour states in ATLAS and CMS
}

\author{
Paolo lengo* \\ CERN, Esplanade des Particules 1, 1211 Meyrin, Switzerland \\ E-mail: paolo.iengodcern.ch
}

\begin{abstract}
Associated production of quarkonia is a key observable for understanding the quarkonium production mechanisms, including the separation of single and double parton scattering components. This talk will present the latest differential measurements from ATLAS and CMS of associatedquarkonium production. In addition, recent results on heavy flavour production measurements are reported, including angular analysis of $B^{0}$ and $B^{+}$decays, searched for tetraquark states and the observation of $Z \rightarrow \psi l^{+} l^{-}$decay.
\end{abstract}

XXVII International Workshop on Deep-Inelastic Scattering and Related Subjects - DIS2019 8-12 April, 2019

Torino, Italy

\footnotetext{
* Speaker.

${ }^{\dagger}$ On behalf of the ATLAS and CMS Collaborations.
} 


\section{Introduction}

The ATLAS and CMS experiments at the Large Hadron Collider offer the possibility to study the production mechanism of quarkonia and heavy flavour (HF) states in a wide range of rapidity and transverse momentum regions and in a range of center-of-mass energies $\sqrt{s}$, as the accelerator has provided collisions at 5, 7,8 and $13 \mathrm{TeV}$ in recent years. Both experiments have analysed data collected in proton-proton $(p p)$, proton-nucleus $(p A)$ and nucleus-nucleus $(A A)$ collisions.

In this paper we report a selection of recent results, including quarkonia production in $p p$ collisions, production of quarkonia pairs, angular analysis of $B^{0} \rightarrow K^{* 0} \mu^{+} \mu^{-}$and $B^{+} \rightarrow K^{+} \mu^{+} \mu^{-}$, searches for tetraquark states decaying into a $B_{s}^{0} \pi$ pair, and observation of $Z \rightarrow \psi l^{+} l^{-}$decay.

Studies of quarkonia and HF states in ATLAS and CMS are based on low $\mathrm{p}_{\mathrm{T}}$ muon trigger and track reconstruction in the tracking detector. The typical transverse momentum resolution for low- $\mathrm{p}_{\mathrm{T}}$ muon tracks is of the order of $1-5 \%$ for both experiments [1] [2].

\section{Quarkonia production}

Final states with oppositely-charged muon pairs are normally selected for these analyses. Production of $J / \psi$ and $\psi(2 S)$ is characterised by the invariant mass and pseudo-proper time distributions of these muon pairs, making it possible to separate the prompt and non-prompt components, the latter coming from B hadron decays in flight. Prompt and non-prompt production mechanisms in $p p$ collisions are described by different models, making the measurement of the differential cross-sections crucial for validating predictions. Figure 1 shows the differential prompt production
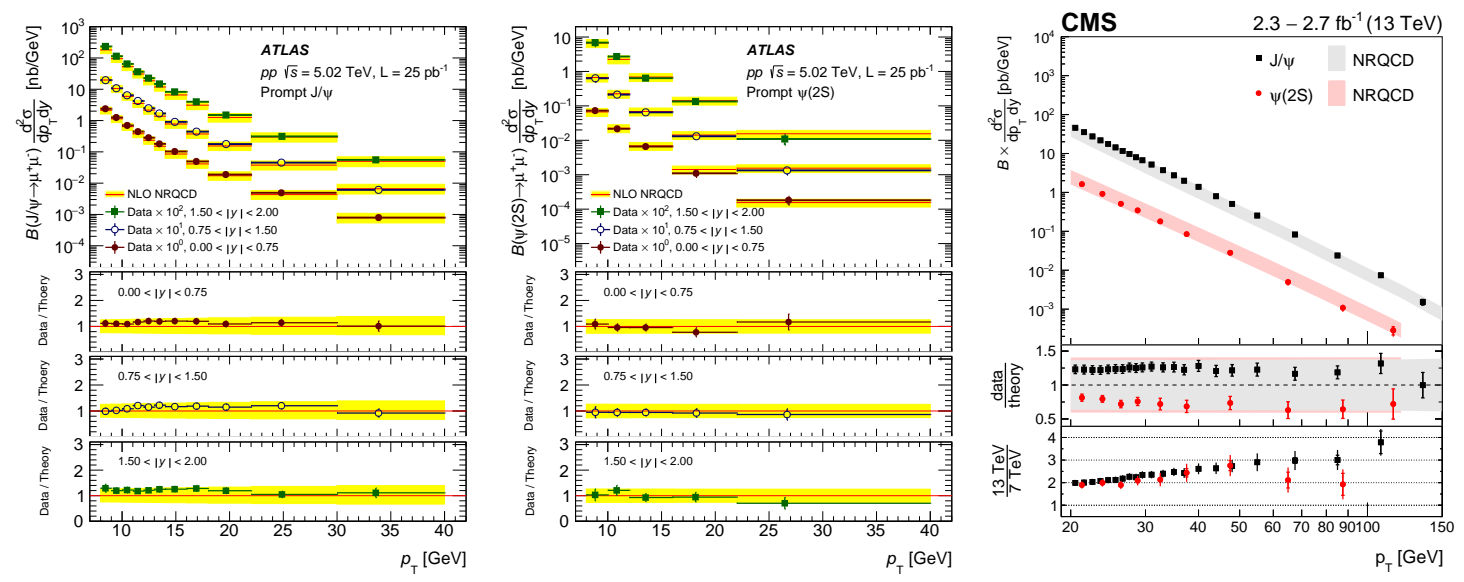

Figure 1: The differential prompt production cross section times di-muon branching fraction of $J / \psi$ and $\psi(2 S)$ as a function of the $\mathrm{p}_{\mathrm{T}}$ of the di-muon system for ATLAS [3] (left and center, respectively) in three intervals of rapidity $y$ in $p p$ collisions at $5.02 \mathrm{TeV}$ and for CMS [4] (right) for $|y|<1.2$ at $13 \mathrm{TeV}$. Statistical and systematic uncertainties are combined in the ATLAS plot, while are shown separately for CMS. The NRQCD theory prediction is also shown.

cross section times di-muon branching fraction of $J / \psi$ and $\psi(2 S)$ as a function of the $\mathrm{p}_{\mathrm{T}}$ of the di-muon system measured by ATLAS [3] with $25 \mathrm{nb}^{-1}$ of data at $5.02 \mathrm{TeV}$ and by CMS [4] with $2.5 \mathrm{fb}^{-1}$ of data at $13 \mathrm{TeV}$. The di-muon decays are assumed to be unpolarised. Good agreement 
with NLO NRQCD [5] calculation is found in the full measured $\mathrm{p}_{\mathrm{T}}$ range at 5.02 and $13 \mathrm{TeV}$, confirming previous measurements at 7 and $8 \mathrm{TeV}$ [6]. Similar measurements for the non-prompt $J / \psi$ and $\psi(2 S)$ are found to be well described by FONLL [7] calculations in the same $\mathrm{p}_{\mathrm{T}}$ range.
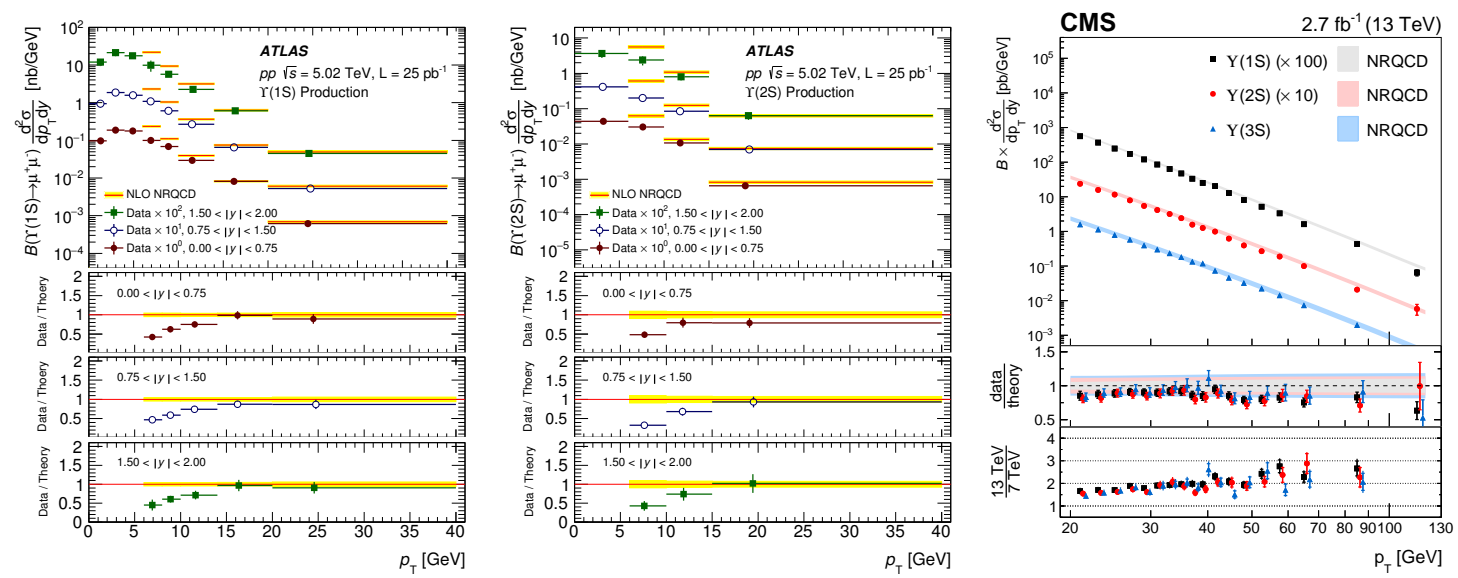

Figure 2: The differential production cross section times di-muon branching fraction of $\Upsilon(\mathrm{nS})$, as a function of the $\mathrm{p}_{\mathrm{T}}$ of the di-muon system measured by ATLAS [3] for three intervals of rapidity $y$ in pp collisions at $5.02 \mathrm{TeV}$ (left and center), and by CMS [4] for $|y|<1.2$ at $13 \mathrm{TeV}$ (right), assuming unpolarised dimuon decays. The vertical error bars correspond to the combined statistical and systematic uncertainties. The NRQCD theory predictions are also shown. The lower panel of the right plot shows the ratios of cross sections measured at $\sqrt{s}=13 \mathrm{TeV}$ to those measured at $7 \mathrm{TeV}$ by CMS [8].

Figure 2 shows the differential production cross section times di-muon branching fraction of the $\Upsilon$ family as a function of $\mathrm{p}_{\mathrm{T}}$ of the di-muon system measured by ATLAS in three intervals of pseudorapidity and by CMS for $|y|<1.2$. Data are compared with NRQCD predictions [8]. There is good agreement between data and calculation for $\mathrm{p}_{\mathrm{T}}>15 \mathrm{GeV}$. For bottomonium production, the long-distance matrix elements, on which the NRQCD calculations are based, are extracted from fitting experimental data only at $\mathrm{p}_{\mathrm{T}}>15 \mathrm{GeV}$. Non-perturbative effects breaking the NRQCD factorisation and perturbative expansion can explain the observed discrepancy at lower $\mathrm{p}_{\mathrm{T}}$.

Quarkonia states can be produced in pairs at LHC, with two different mechanisms: single parton scattering (SPS) where the two states are produced in a single interaction between partons; and double-parton scattering (DPS) where simultaneous, independent and uncorrelated interactions of two pairs of partons take place, each one producing a quarkonium. The process is described by the effective cross section, sensitive to the spatial distribution of gluons in the protons:

$$
\sigma_{\text {eff }}=\frac{1}{2} \frac{\sigma_{\mathrm{J} / \psi}^{2}}{\sigma_{\mathrm{DPS}}^{\mathrm{J} / \psi \mathrm{J} / \psi}}=\frac{1}{2} \frac{\sigma_{\mathrm{J} / \psi}^{2}}{\mathrm{f}_{\mathrm{DPS}} \times \sigma_{\mathrm{J} / \psi \mathrm{J} / \psi}}
$$

where $f_{\text {DPS }}$ is the fraction of DPS events. The value of $\sigma_{\text {eff }}$ is expected to be in the range (2-20) $\mathrm{mb}$ and assumed to be independent of the scattering process and $\sqrt{s}$. ATLAS has measured the effective cross section of di-J $/ \psi$ production analysing $11.8 \mathrm{fb}^{-1}$ at $8 \mathrm{TeV}$ collision data [9]. Data driven estimations of SPS contribution, based on angular distributions, have been used. The reconstructed number of di $-J / \psi$ events from a fit procedure is $3310 \pm 330$ and $3140 \pm 370$ for pseudorapidity intervals of $|y|<1.05$ and $1.05<|y|<2.1$, respectively. The measured DPS fraction is $\mathrm{f}_{\text {DPS }}=$ 
$(9.2 \pm 2.1$ (stat) \pm 0.5 (syst) $) \%$ and the effective DPS cross section $\sigma_{\text {eff }}=6.3 \pm 1.6$ (stat $) \pm 1.0$ (syst) mb. A comparison of measurements performed by UA2, D0, CDF, ATLAS, CMS and LHCb Collaborations [9] suggests that $\sigma_{\text {eff }}$ is generally lower for prompt di- $J / \psi$ and di- $\Upsilon$ with respect to other final states (for example with $J / \psi$ associated with vector bosons) by a factor $\sim 3$. Quarkonium final states are dominantly produced from gluon-gluon interactions, while channels with jets mostly by quark-antiquark and quark-gluon parton interactions. The observed trend could be related to smaller average transverse distance between gluons in the proton than between quarks or quarks and gluons. More precise measurements are needed for a definitive statement on this trend. The trend is qualitatively confirmed by the measurement from CMS of $\Upsilon(1 \mathrm{~S})$ pair production, with a dataset of $20.7 \mathrm{fb}^{-1}$ at $8 \mathrm{TeV}[10]$. The number of reconstructed di- $\Upsilon(1 \mathrm{~S})$ events (38 \pm 7 at $|y|<2.0$ ) translates to an effective cross section of $\sigma_{\text {eff }} \approx 2.2-6.6 \mathrm{mb}$, assuming isotropically decaying $\Upsilon$.

\section{Open Beauty production}

Both ATLAS and CMS have performed the angular analysis of the $B^{0} \rightarrow K^{* 0} \mu^{+} \mu^{-}$decay, with the $K^{* 0}$ decaying into a $K^{+} \pi^{-}$pair. The decay is particularly interesting as it is a FCNC process forbidden at tree level. It can be fully described by three kinematic angles and the dimuon invariant mass squared $q^{2}$. New physics entering the loop can be detected by looking at the angular differential decay rate; generally expressed in terms of $\mathrm{P}_{\mathrm{i}}$ and $\mathrm{P}_{\mathrm{j}}^{\prime}$ observables built up as combination of forward-backward asymmetry $\left(\mathrm{A}_{\mathrm{FB}}\right)$, fraction of longitudinally polarised $K^{*}\left(\mathrm{~F}_{\mathrm{L}}\right)$, and s-wave fraction $F_{S}$. Both experiments have analysed a dataset of about $20 \mathrm{fb}^{-1}$ of $8 \mathrm{TeV}$ collision data collected in 2017. ATLAS [11] extracts $\mathrm{P}_{1}$ and $\mathrm{P}_{\mathrm{i}}^{\prime}(\mathrm{i}=4,5,6,8)$ with four different fits to signal and background distributions, fitting 340 events in $3 q^{2}$ bins. CMS [12] extracts $\mathrm{P}_{1}$ and $\mathrm{P}_{5}^{\prime}$ with a fit to signal and background distributions, including the mistagging probability for signal events, selecting 1400 events in seven $q^{2}$ bins.

Experimental results are compared with three models: DHMV [13] and JC [14] which include QCD factorisation with hadronic uncertainties taken from calculation; and CFFMPSV [15] where contributions of hadronic charm are fitted from data. ATLAS finds general good agreement with SM expectation in all bins for the four fitted parameters, except a $\sim 2.5 \sigma$ deviation from DHVM for $\mathrm{P}_{4}^{\prime}$ (Fig. 3 left) and $\mathrm{P}_{5}^{\prime}$ (Fig. 3 center) in one bin. LHCb sees a notable $>3 \sigma$ discrepancy on $\mathrm{P}_{5}^{\prime}[16]$. CMS data are compatible with the SM prediction in the whole range, favouring DHMV model at low $q^{2}$ (Fig. 3 right).

The CMS Collaboration has recently performed an angular analysis of the $B^{+} \rightarrow K^{+} \mu^{+} \mu^{-}$ decay [18] with $20.5 \mathrm{fb}^{-1}$ at $8 \mathrm{TeV}$ data. The decay rate depends on the angle $\theta_{\mathrm{L}}$ between the direction of $\mu^{-}$and $K^{+}$in the di-muon rest frame. The rate is expressed in terms of forwardbackward asymmetry $\mathrm{A}_{\mathrm{FB}}$, and the contribution from the pseudoscalar, scalar and tensor amplitudes to the decay width $\mathrm{F}_{\mathrm{L}}$. A 2D unbinned max-likelihood fit to $\mathrm{M}(K \mu \mu)$ and $\cos \theta_{\mathrm{L}}$ distributions is repeated in seven $q^{2}$ bins in the interval (1-22) $\mathrm{GeV}^{2}$, selecting a total of $2286 \pm 73$ events. The measured values of $\mathrm{A}_{\mathrm{FB}}$ and $\mathrm{F}_{\mathrm{L}}$ are compatible with $\mathrm{SM}$ predictions and are consistent with previous measurements.

In the search for exotic hadrons, a special case is represented by the $X(5568)$ state decaying into a $B_{s}^{0} \pi^{ \pm}$pair. The observation of a narrow resonance with a mass of $5568 \mathrm{MeV}$ and a width of $21 \mathrm{MeV}$ was published by the D0 Collaboration in 2016 [19] and confirmed in 2018 [20]. 

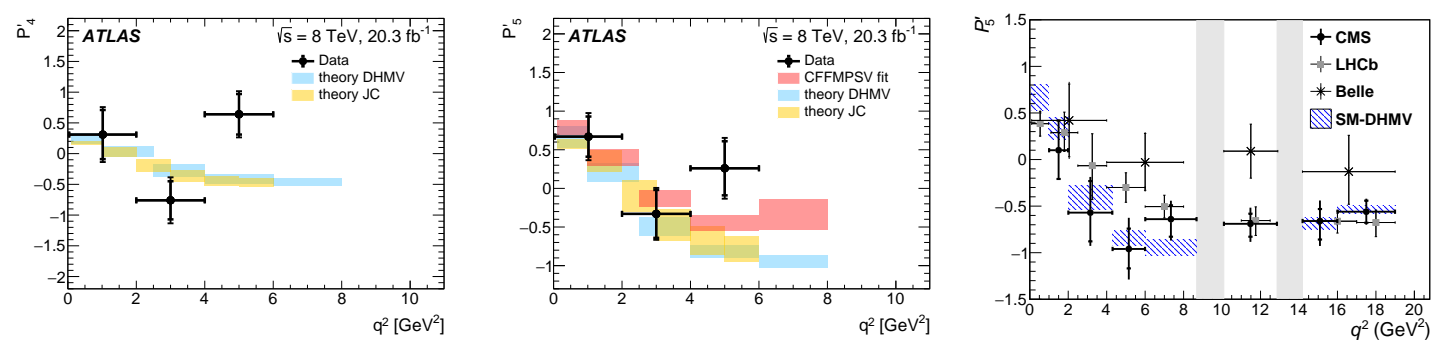

Figure 3: Measured values of $\mathrm{P}_{4}^{\prime}$ (left) and $\mathrm{P}_{5}^{\prime}$ (center) by ATLAS [11] and $\mathrm{P}_{5}^{\prime}$ (right) by CMS [12] for the $B^{0} \rightarrow K^{* 0} \mu^{+} \mu^{-}$decay, compared with predictions from the theoretical calculations. The CMS plot includes the comparison with the results from the LHCb [16] and Bellle [17] Collaborations. In all plots, statistical and total uncertainties are shown for the data.

Subsequent searches performed by the LHCb [21] and CDF [22] Collaborations did not confirm the observation, setting instead an upper limit on $\rho_{\mathrm{X}}$, the cross-section ratio of $X(5568)$ to $B_{s}^{0}$. Searches for $X^{ \pm} \rightarrow B_{s}^{0} \pi^{ \pm}$have been carried out by ATLAS with $4.9 \mathrm{fb}^{-1}$ of $7 \mathrm{TeV}$ and $19.5 \mathrm{fb}^{-1}$ of $8 \mathrm{TeV}$ data [23] and by CMS with $19.7 \mathrm{fb}^{-1}$ of $8 \mathrm{TeV}$ data [24]. No signal evidence has been found either by ATLAS or by CMS. Upper limits on $\rho_{\mathrm{X}}$ have been set in the mass range $5550<\mathrm{M}\left(B_{s}^{0} \pi^{+}\right)<5700$ $\mathrm{MeV}$ (ATLAS, Fig 4 left) and $5550<\mathrm{M}\left(B_{s}^{0} \pi^{+}\right)<5900 \mathrm{MeV}$ (CMS, Fig 4 right), which are at present the most stringent limits on this parameter. In particular CMS has $\rho_{\mathrm{X}}<1.1 \%$ at $95 \%$ C.L. for $\mathrm{p}_{\mathrm{T}}\left(B_{s}^{0}\right)>10 \mathrm{GeV}$ for a $X$ state of a mass $5568 \mathrm{MeV}$.
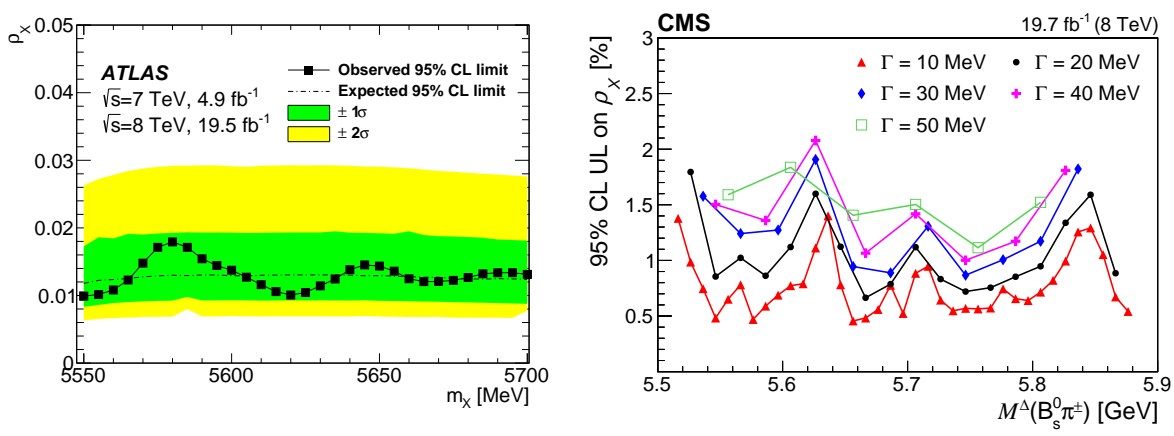

Figure 4: The $95 \%$ C.L. upper limit on $\rho_{\mathrm{X}}$ as a function of the mass of a possible exotic state decaying into $B_{s}^{0} \pi^{ \pm}$measured by ATLAS [23] (left) for a fixed value of the natural width of the state of $21 \mathrm{MeV}$, and by CMS [24] (right), for five different values of the width. The ATLAS plot includes the \pm 1 and $\pm 2 \sigma$ bands.

\section{Observation of $Z \rightarrow \psi l^{+} l^{-}$}

CMS has recently observed the $Z \rightarrow \psi l^{+} l^{-}$decay [25], where $\psi$ indicated either direct $J / \psi$ or indirect $J / \psi$ from $\psi(2 S)$ decay, with $35.9 \mathrm{fb}^{-1}$ collision data at $13 \mathrm{TeV}$. The $J / \psi$ is detected via its decay into a muon pair, thus the final state of the $Z$ decay contains either $4-\mu$ or $2-\mu$ and 2-e. The fit to the selected candidate events gives the following yields: $13.0 \pm 3.9$ and $11.2 \pm 3.4$ for the $\mu$ and the $e$ channel, respectively. The combined significance is $>5 \sigma$. After subtraction of the indirect contribution, the ratio of the branching fraction of the exclusive decay $Z \rightarrow J / \psi l^{+} l^{-}$to the decay $Z \rightarrow \mu^{+} \mu^{-} \mu^{+} \mu^{-}$within a fiducial phase space is measured to be $0.67 \pm 0.18$ (stat) \pm 0.05 (syst). 


\section{Conclusion}

The ATLAS and CMS Collaborations have a rich physics at the LHC program for studies of quarkonia and heavy flavour physics. Measurements of production cross-sections are crucial for validating models, while measurements of kinematic parameters predicted by calculation could reveal beyond-SM contributions. A selection of recent results have been reported, including quarkonia production, angular analysis of $B^{0} \rightarrow K^{* 0} \mu^{+} \mu^{-}$and $B^{+} \rightarrow K+\mu^{+} \mu^{-}$decays, searches for resonances decaying into $B_{s}^{0} \pi^{ \pm}$and the observation of $Z \rightarrow \psi l^{+} l^{-}$decay. No evidence of BSM effects has been measured, with ATLAS reporting a tension of $\sim 2.5 \sigma$ on $\mathrm{P}_{4}^{\prime}$ and $\mathrm{P}_{5}^{\prime}$ parameters for the $B^{0} \rightarrow K^{* 0} \mu^{+} \mu^{-}$decay. Also, no evidence of a tetraquark state decaying into $B_{s}^{0} \pi^{ \pm}$has been observed.

\section{References}

[1] ATLAS Collaboration, 2008 JINST 3 S08003

[2] CMS Collaboration, JINST 3 S08004

[3] ATLAS Collaboration, Eur. Phys. J. C (2018) 78, 171

[4] CMS Collaboration, Phys. Lett. B 780, 251

[5] Y. Ma, K. Wang and K. Chao Phys. Rev. Lett. 106 (2011), 042002; H. Shao et. al JHEP 05 (2015), 103

[6] M. Cacciari et. al JHEP 10 (2012), 137

[7] ATLAS Collaboration, Eur. Phys. J. C (2016) 76, 283

[8] H. Han et. al Phys. Rev. D 94 (2016), 014028

[9] ATLAS Collaboration, Eur. Phys. J. C (2017) 77, 76

[10] CMS Collaboration, JHEP 05 (2017), 013

[11] ATLAS Collaboration, JHEP 10 (2018), 047

[12] CMS Collaboration, Phys. Lett. B 781, 517

[13] S. Descotes-Genon, L. Hofer, J. Matias and J. Virto, JHEP 12 (2014) 125

[14] S. Jager and J. Martin Camalich, Phys. Rev. D 93 (2016) 014028

[15] M. Chiuchini et. al JHEP 06 (2016) 116

[16] LHCb Collaboration, JHEP 02 (2016), 104

[17] Belle Collaboration, Phys. Rev. Lett. 118 (2017), 111801

[18] CMS Collaboration, Phys. Rev. D 98, 112011

[19] D0 Collaboration, Phys. Rev. Lett. 117, 022003

[20] D0 Collaboration, Phys. Rev. D 97, 092004

[21] LHCb Collaboration, Phys. Rev. Lett. 117, 152003

[22] CDF Collaboration, Phys. Rev. Lett. 120 (2018), 202006

[23] ATLAS Collaboration, Phys. Rev. Lett. 120 (2018), 202007

[24] CMS Collaboration, Phys. Rev. Lett. 120 (2018), 202005

[25] CMS Collaboration, Phys. Rev. Lett. 121, 141801 